\title{
Development of critical pathway for ABO incompatible kidney transplant patients
}

\author{
Heeyoung Kim, Manki Ju, Jung Jun Lee, Sunyoung Son
}

Department of Surgery-Transplantation, Gangnam Severance Hospital, Seoul, Korea

Background: A critical pathway (CP), a part of case management, has emerged in the health care delivery system for quality management that can satisfy the needs of patients with limited manpower and time and increase the satisfaction of medical personnel. In the case of $A B O$ incompatible transplantation, the complex process that requires preoperative treatment such as plasmapheresis and immunosuppressant treatment and additional postoperative treatment and monitoring, and communication of a multidisciplinary team in the process, is required. If suitable CP is developed, it is expected that the quality of the treatment process and efficient operation will be achieved. This study was conducted to develop CP for ABO incompatible kidney transplant patients at one hospital.

Methods: The research method forms a conceptual frame of reference based on the literature review and the protocol currently used in this hospital, and is preliminarily prepared through meetings between multidisciplinary teams such as transplant surgery, nephrology, diagnostic laboratory medicine, radiology, blood bank, and nursing. In order to prepare the CP and confirm its practical applicability, the final CP is confirmed after verifying the clinical validity for kidney transplantation patients from August to September 2021.

Results: In the $\mathrm{CP}$, eight items including examination, drug, treatment, diet, exercise, assessment, patient and caregiver education on the vertical axis and the horizontal axis were determined from the time of admission to the day of discharge.

Conclusions: By applying the $\mathrm{CP}$ developed as a result of this study to practice, it is expected that the $\mathrm{ABO}$ incompatible kidney transplantation process will be standardized to improve the quality of medical care and improve the efficiency of medical institution operation.

Corresponding author: Heeyoung Kim

E-mail: amelli@yuhs.ac

(c) The Korean Society for Transplantation

This is an Open Access article distributed under the terms of the Creative Commons Attribution Non-Commercial License (http://creativecommons.org/licenses/by-nc/4.0/) which permits unrestricted non-commercial use, distribution, and reproduction in any medium, provided the original work is properly cited. 